\title{
Article \\ Selection of Lactic Acid Bacteria Species and Strains for Efficient Trapping of Drosophila suzukii
}

\author{
Amani Alawamleh ${ }^{1,2}{ }^{\mathbb{D}}$, Gordana Đurović ${ }^{2,3}$, Giuseppe Maddalena ${ }^{1}$, Raffaele Guzzon ${ }^{4}$, Sonia Ganassi ${ }^{1}{ }^{1}$, \\ Maaz Maqsood Hashmi ${ }^{5}$ (D), Felix Wäckers ${ }^{2}$, Gianfranco Anfora ${ }^{3,6, *(D)}$ and Antonio De Cristofaro ${ }^{1}$ (D) \\ 1 Department of Agricultural, Environmental and Food Sciences, University of Molise, Via De Sanctis, \\ 86100 Campobasso, Italy; amaniawamleh@yahoo.com (A.A.); peppemad@hotmail.com (G.M.); \\ sonia.ganassi@gmail.com (S.G.); decrist@unimol.it (A.D.C.) \\ 2 Biobest Group NV, Ilse Velden, 2260 Westerlo, Belgium; valerianaof@gmail.com (G.Đ.); \\ Felix.Waeckers@biobestgroup.com (F.W.) \\ 3 Research and Innovation Centre, Fondazione Edmund Mach, Via Edmund Mach 1, 38098 San Michele \\ all'Adige, Italy \\ 4 Technology Transfer Centre, Fondazione Edmund Mach, 38098 San Michele all'Adige, Italy; \\ raffaele.guzzon@fmach.it \\ 5 Consiglio Nazionale Della Ricerche, Instituto di Ricerca Sugli Ecosistemi Terrestri, Via Guglielmo Marconi 2, \\ 05010 Porano, Italy; maazmh@hotmail.com \\ 6 Centre Agriculture Food Environment (C3A), University of Trento, Via Edmund Mach 1, 38098 San Michele \\ all'Adige, Italy \\ * Correspondence: Gianfranco.anfora@fmach.it
}

check for updates

Citation: Alawamleh, A.; Đurović, G.; Maddalena, G.; Guzzon, R.; Ganassi, S.; Hashmi, M.M.; Wäckers, F.; Anfora, G.; Cristofaro, A.D. Selection of Lactic Acid Bacteria Species and Strains for Efficient Trapping of Drosophila suzukii. Insects 2021, 12, 153. https://doi.org/ $10.3390 /$ insects 12020153

Academic Editors: Christos G. Athanassiou and Eric W. Riddick Received: 13 November 2020 Accepted: 8 February 2021 Published: 11 February 2021

Publisher's Note: MDPI stays neutral with regard to jurisdictional claims in published maps and institutional affiliations.

Copyright: (C) 2021 by the authors Licensee MDPI, Basel, Switzerland. This article is an open access article distributed under the terms and conditions of the Creative Commons Attribution (CC BY) license (https:// creativecommons.org/licenses/by/ $4.0 /)$.
Simple Summary: The spotted wing drosophila (SWD) is an invasive fruit fly that causes serious economic damage to many fruit crops. Monitoring is the first step for any management program to determine the characteristics of a pest. For this purpose, there are no efficient baits registered to date. Certain bacteria release chemical compounds that attract SWD. We studied the bacterial impact on the enhanced attractiveness of a commercial bait (Droskidrink ${ }^{\circledR}$ ) under field and laboratory conditions. At first, Oenococcus oeni belonging to lactic acid bacteria (LAB) was found to release chemical compounds that were highly attractive for SWD. The attractiveness of Droskidrink ${ }^{\circledR}$ bait was increased by O. oeni culture, resulting in a higher capture rate of SWD in traps. Therefore, our findings suggest the use of the bacterial culture inside the commercial SWD baits. The use of these kinds of baits can minimize the risk of pest outbreaks in fruit orchards in both domestic and wild environments. Our pest management approach is farmer-friendly in all aspects, as well as the food sector.

Abstract: (1) Monitoring of Drosophila suzukii is based on the use of effective traps and baits. The current baits are insufficient to provide efficient monitoring. The use of bacteria as bio-catalyzers to produce bioactive volatiles may improve flies' attraction. Thus, we conducted this work to improve Droskidrink ${ }^{\circledR}$ bait's attractiveness using lactic acid bacteria. (2) Different baits that were based on the use of Droskidrink ${ }^{\circledR}$ were assessed for flies' attraction in a Droso-Trap ${ }^{\circledR}$ in a vineyard. Oenococcus oeni, Pediococcus spp., and Lactobacillus spp. were used. The performance of the most attractive species, $O$. oeni, inoculated into Droskidrink ${ }^{\circledR}$ was assessed in laboratory tests. The responses of female flies to volatiles produced by Droskidrink ${ }^{\circledR}$ with O. oeni strains were recorded by electroantennography. (3) Preliminary field assessment of baits recorded O. oeni as the most attractive species. Three strain groups showed adaptation to test conditions. Volatiles extracted by the headspace of baits inoculated with O. oeni, elicited electroantennographic responses from fly antennae. (4) Droskidrink ${ }^{\circledR}$ inoculated with O. oeni is a highly attractive bait for monitoring. These findings will be useful for improving the attractiveness of D. suzukii commercial baits based on the utilization of LAB volatiles in a strain-dependent manner.

Keywords: lactic acid bacteria; Oenococcus; food bait; spotted wing drosophila 


\section{Introduction}

Drosophila suzukii Matsumura (Diptera: Drosophilidae) is an invasive fruit fly of Asian origin that rapidly invaded Europe, causing great losses in soft fruit production [1-4]. The rapid invasive spread of $D$. suzukii across Europe expanded to temperate/cool climates, causing severe damage to fruit crops and posing an economic threat to the small soft fruit industries [3]. Fruit damage is caused by larva feeding and development within the infested fruits, making them non-saleable [4]. D. suzukii is a highly polyphagous pest that infests a broad range of soft-skinned fruits, i.e., berry fruits blackberry, raspberry, blueberry, strawberry, and grapes [2].

The management of targeted pests is mainly implemented with conventional chemical insecticides. Among the registered insecticides, organophosphates, spinosyns and pyrethrins, in timely applications, can provide an adequate level of control [5-7]. At present, chemical control methods often show limitations and failures due to pest population density, and the specific social and agronomical context. Among environmentally safe approaches, those based on the interferences with insect communication often provide efficient control, i.e., mass trapping and attract and kill [8,9]. On the other hand, D. suzukii populations could be reduced by exploiting biocontrol agents (fungi, bacteria, and viruses) and other natural enemies of this pest, such as parasitoids and predators [10-13]. However, the evaluation of these control methods in the laboratory and in field assays and their development for commercial use are still ongoing.

Therefore, D. suzukii monitoring, as a part of pest management, is the key to controlling this pest. It assists in the detection of D. suzukii in host crops and provides information about feeding patterns, behavior throughout the year, and population dynamics. Hence, it is important to use the best available traps and baits to obtain the most adequate monitoring. Primarily, D. suzukii population monitoring is being implemented by using differentially shaped and colored traps baited with fermentation products such as apple cider vinegar, wine, or yeast/sugar as attractive lures [14-21]. At present, fermentation products are not adequately selective and effective for D. suzukii monitoring [20], and no efficient monitoring tools have been developed yet. Despite that, it has been demonstrated that the attractiveness of food baits can be increased by the addition of different bacterial species. In this regard, the investigation of volatile organic compounds produced by bacterial fermentation identified the key compounds for effective fly attraction. Such attractiveness has been investigated in behavioural bioassays, along with the chemical characterization of the volatiles profile. Strains and species of symbiotic acetic acid bacteria commonly found in D. suzukii Italian populations (Acetobacter, Gluconobacter, and Komagataeibacter) were tested with an olfactometer. Female flies showed a significant attraction to some strains of Gluconobacter and Komagataeibacter species, whose volatile compounds were proposed as a useful tool for developing sustainable control strategies [22].

Lactic acid bacteria (LAB) are widespread microorganisms which can be found in any environment rich in carbohydrates, such as plants and fermented foods. They are Gram-positive and acid-tolerant microorganisms that grow anaerobically. LAB include several species, i.e., the genus Lactobacillus, as well as the genera Pediococcus, Leuconostoc, Streptococcus, and Oenococcus. The fast-growing characteristics of LAB strains and their metabolic activities are the key to LAB's benefits and applications [23].

The primary LAB metabolic activity is the degradation of carbohydrates into different compounds, mainly lactic acid through the fermentation process [24]. However, they share several unique biochemical characteristics when compared to other bacterial groups (e.g., high malolactic activity, production of volatile compounds, growth rate) [25]. Oenococcus oeni (Garvie) is the species that best adapts to the harsh wine conditions, due to its ability to tolerate low $\mathrm{pH}$ and high concentrations of ethanol and sulfites [26]. These LAB characteristics distinguish them from other bacterial species, making them good candidates for a more attractive and selective food bait.

The LAB's ability to produce bioactive volatiles attracting fruit Drosophila flies was evaluated in previous studies. The L. brevis and L. plantarum species were identified from 
Drosophila melanogaster (Meigen) larval gut, and their emitted volatile compounds were evaluated for fly attraction. Moreover, larval and adult flies showed a significant attraction to volatiles emanating from food substrates that have been occupied by larvae, indicating that flies rely on microbial volatiles for long distance attraction to suitable feeding sites [27].

Additionally, D. melanogaster exhibited behavioral preferences towards gut microbes. Both adults and larvae were attracted to volatile compounds associated with the fly microbiome, i.e., L. plantarum in choice assays [28]. Similarly, symbiotic LAB species were identified in D. suzukii gut, i.e., L. plantarum and L. brevis [29]. D. suzukii attraction towards volatiles emitted by LAB species has not been assessed yet. There is a need to investigate the use of LAB species as a source of attraction for D. suzukii flies and their subsequent potential application as components of a more attractive food bait.

The current study attempts to fill numerous gaps in knowledge regarding the attractive effect of volatiles emitted by LAB species on $D$. suzukii fly preferences which will be helpful for developing effective tools for $D$. suzukii monitoring. Therefore, we decided to improve the attractiveness of commercially available Droskidrink ${ }^{\circledR}$ food bait using active cultures of LAB species. In this study, we aimed to evaluate the attractiveness of different LAB species and strains for $D$. suzukii flies under field and laboratory conditions. The data obtained contribute to improving the food baits that are currently used to monitor D. suzukii and to developing new tools for efficient pest management, focusing on the exploitation of bioactive volatiles emitted by LAB strains.

\section{Materials and Methods}

\subsection{Field Assessment of the Attractiveness of Droskidrink ${ }^{\circledR}$ Food Baits Inoculated with Different Lactic Acid Bacteria}

A preliminary field experiment to compare the attractiveness of different food baits for $D$. suzukii flies was conducted in a commercial vineyard var. Teroldego in San Michele all'Adige, Trento, Italy. The commercial food bait Droskidrink ${ }^{\circledR}$ (DD) (Prantil, Trento, Italy) was used in all bait types. DD comprises a mixture of apple cider vinegar $(75 \%)$, red wine $(25 \%)$, and $20 \mathrm{~g} / \mathrm{L}$ of brown cane sugar [30]. The tested LAB including Oenococcus oeni, Lactobacillus spp., and Pediococcus spp. were selected from the bacterial culture collection of Fondazione Edmund Mach laboratories, San Michele all'Adige, Italy and inoculated in the commercial food bait.

The species were previously isolated from traditional Italian wine during the early phase of malolactic fermentation and identified based on gene sequencing [31]. LAB species were used as catalyzers of the production of bioactive compounds to D. suzukii, and their performance was assessed based on the biochemical changes in the DD bait. In this experiment, 10 types of food bait were used with different compositions at different $\mathrm{pH}$ values, as indicated in Table 1.

Droso-Trap ${ }^{\circledR}$ (Biobest, Westerlo, Belgium) was deployed for field trapping. In brief, the trap designed for trapping Drosophila flies consists of a transparent lid with a wire hanger and a red plastic base containing three inlet tubes, each with seven holes with a $5 \mathrm{~mm}$ diameter for fly entry. The $\mathrm{pH}$ of the baits inoculated with bacterial strains was adjusted to 4.0 to ensure adequate bacterial growth. A drowning solution of DD was placed within the trap cup. Each trap contained $200 \mathrm{~mL}$ of liquid bait and a drop of Triton ${ }^{\circledR}$ X-100 (Sigma-Aldrich Co., St. Louis, MO, USA) to reduce the liquid bait surface tension and promote the capture and submerging of flies.

Bait type $\mathrm{H}$ was pasteurized to inhibit any microbial growth in the liquid bait. Bait type I was mixed with $10 \mathrm{~mL} / \mathrm{L}$ of cycloheximide solution $0.01 \%$ (Oxoid, Cheshire, UK) to prevent yeast contamination. Bait type $\mathrm{L}$ was mixed with tetracycline (antibiotic) to inhibit bacterial growth in the liquid bait. The nominal concentration of bacterial species inoculated into each bait was adjusted to $10^{6} \mathrm{CFU} / \mathrm{mL}$. The traps were placed in shady spots, $1 \mathrm{~m}$ above the ground at the level of the grapes. The randomized complete block design was applied in three different blocks with three replications each (Figure S1). All traps were maintained in the field for a seven-week period and checked weekly. Liquid baits were 
examined in the laboratory, captures of $D$. suzukii flies were recorded, and the liquid bait was replaced once a week. The numbers of $D$. suzukii flies were analyzed with a Poisson generalized linear model (GLM) [32].

Table 1. Composition of Droskidrink ${ }^{\circledR}$ food baits tested in a preliminary field experiment in a vineyard var. Teroldego in San Michele all'Adige, Trento, Italy.

\begin{tabular}{|c|c|}
\hline Type & Composition \\
\hline Bait A & $\begin{array}{l}\text { DD (pH 4.0) inoculated with Oenococcus oeni } \\
\text { ATCCBAA-331 }\end{array}$ \\
\hline Bait B & DD (pH 4.0) inoculated with Pediococcus spp. \\
\hline Bait C & $\begin{array}{l}\text { DD (pH 4.0) inoculated with Lactobacillus spp. } \\
\text { V223 }\end{array}$ \\
\hline Bait D & $\begin{array}{l}\text { DD (pH 4.0) inoculated with Lactobacillus spp. } \\
\text { V313 }\end{array}$ \\
\hline Bait E & $\begin{array}{l}\text { DD (pH 4.0) inoculated with Lactobacillus spp. } \\
\text { L308 }\end{array}$ \\
\hline Bait F & DD at $\mathrm{pH} 4.0$ \\
\hline Bait G & DD at pH 2.5 (commercial version) \\
\hline Bait $\mathbf{H}$ & $\begin{array}{l}\mathrm{DD} \text { at } \mathrm{pH} 2.5 \text { pasteurized at } 70^{\circ} \mathrm{C} \text { for } 30 \mathrm{~min} \\
\text { and inoculated with Oenococcus oeni }\end{array}$ \\
\hline Bait I & $\begin{array}{c}\mathrm{DD} \text { at } \mathrm{pH} 4.0 \text { pasteurized at } 70^{\circ} \mathrm{C} \text { for } 30 \mathrm{~min}, \\
\text { mixed with } 10 \mathrm{~mL} / \mathrm{L} \text { of cycloheximide } \\
\text { aqueous solution }(0.01 \%) \text {, and inoculated with } \\
\text { Oenococcus oeni }\end{array}$ \\
\hline Bait L & $\begin{array}{l}\mathrm{DD} \text { at } \mathrm{pH} 4.0 \text { mixed with } 1 \mathrm{~g} \text { of tetracycline } \\
\text { and inoculated with Oenococcus oeni }\end{array}$ \\
\hline
\end{tabular}

The experiment included three trials that were conducted sequentially during the summer of 2013, using a different number of treatments in each trial. Each trial comprised three replicates where 6,7 , and 10 sample traps were placed in the first, second, and third trial, respectively. All trials were carried out in the same vineyard, adjacent to the forest area. The first trial focused on comparing DD baits inoculated with LAB strains i.e., A, B, $\mathrm{C}, \mathrm{D}, \mathrm{E}$, and $\mathrm{F}$ baits. In the second trial, we focused on comparing the first six tested baits against the commercial version of DD (bait type G). Finally, we compared all previously tested baits with DD baits of different compositions, i.e., $\mathrm{H}, \mathrm{I}$, and L.

\subsection{Assessment of The Performance of Oenococcus oeni Strains in Droskidrink ${ }^{\circledR}$ Food Bait}

Based on the findings of the preliminary field experiment (see Results section), $O$. oeni was selected as the most suitable bio-catalyzer to improve the attractiveness of DD. Therefore, fourteen strains of $O$. oeni were tested to determine their viability and fermentative metabolism in a standard medium for LAB. Strains isolated from Italian wines were obtained from the bacterial culture collection of Fondazione Edmund Mach laboratories, San Michele all'Adige, Italy. The selected strains showed high resistance to wine-limiting factors (low $\mathrm{pH}$, high ethanol concentration, and low fermentation temperature) and performed a reliable malolactic fermentation in previous work [33].

All strains were cultured in modified MRS broth medium (MRSm) (Oxoid, Milan, Italy) in a 96 micro volume $(200 \mu \mathrm{L})$ plate (Starstedt, Germany) at $25^{\circ} \mathrm{C}$. Thereafter, the MRSm was used to assay the bacterial growth at the main values of the Droskidrink ${ }^{\circledR}$ parameters $(\mathrm{pH}$ value 4.0 , acetic acid concentration $45 \mathrm{~g} / \mathrm{L}$, ethanol content $4 \%(v / v))$ that would act as limiting factors for the metabolic performance of O. oeni strains. In this case, strains were tested at $\mathrm{pH}$ value 4.0 , acetic acid concentration $45 \mathrm{~g} / \mathrm{L}$, ethanol content $4 \%(v / v)$, and incubation temperature $15^{\circ} \mathrm{C}$, all likely encountered during the field 
trapping. In each test, one DD parameter value was assessed with standard values of other parameters. In this experiment, three technical replicates and three biological replicates were used. Optical density (OD) measurements of bacterial cultures were performed in a PowerWave HT Microplate Spectrometer (BioTek, Winooski, VT, USA) with a Costar Flat Bottom 96-well plate with lid and $200 \mu \mathrm{L}$ per well. Absorbance was measured at wavelength $480 \mathrm{~nm}$ and temperature $25^{\circ} \mathrm{C}$ and the mean of three readings was taken. The increase in OD at $480 \mathrm{~nm}$ was measured. The OD at $480 \mathrm{~nm}$ of MRSm was used as blank. The incubation time was adjusted according to the methods recommended by the International Organization of Viticulture and Wine (OIV).

\subsection{Electroantennography Responses of Drosophila suzukii Females to Volatile Collection of Oenococcus oeni Strains in Droskidrink ${ }^{\circledR}$ Food Bait}

On the basis of the assessment of the performance of fourteen O. oeni strains in DD bait (see Results section), three strains were selected and used to determine the electrophysiological response of $D$. suzukii flies to volatile compounds released during the fermentation process. Then, the antennal activity responses of mated females $(n=5)$ to the volatile compounds released by the three selected O. oeni strains (strain 2 , strain 12 , and strain 13) and the reference strain MRI 10000 were recorded by using a standard electroantennography (EAG) apparatus (Syntech).

Experimental flies were collected from a laboratory colony that had already been established from collected fly populations in Trento Province, Italy. The colony was reared on a standard cornmeal-based artificial diet and maintained at $24 \pm 1{ }^{\circ} \mathrm{C}, 65 \pm 5 \%$ relative humidity and (16:8 L:D) photoperiod. Volatiles were collected with the closed loop stripping approach (CLSA) [34]. A $50 \mathrm{~mL}$ DD sample was poured into a glass jar with a plastic lid with two small openings. Both openings were connected with tubes in a circuit through the jar, where one tube was connected to a CLSA carbon filter (Brechbühler AG, Schlieren, Switzerland) while the other one was connected to the miniature $12 \mathrm{~V}$ vacuum graphite pump (Fürgut $\mathrm{GmbH}$, Tannheim, Germany) to circulate the air through the jar. After 60 min of air flux, volatiles concentrated in the CLSA filter were eluted with $100 \mu \mathrm{L}$ of dichloromethane (J.T. Baker, Deventer, Holland).

Each eluted sample represented a stimulus. Each stimulus was prepared by adsorbing aliquots of the solution $(25 \mu \mathrm{L})$ on filter paper $1.5 \mathrm{~cm}^{2}$ and inserting them into a Pasteur pipette. The Pasteur pipettes were closed on the thinner side with a $1 \mathrm{~mL}$ blue tip. The solvent was allowed to evaporate for $10 \mathrm{~min}$ before starting the experiment. Three control pipettes, including an empty pipette, a pipette filled with paraffin oil solvent, and a pipette filled with dichloromethane solvent, served as blanks. Before and after each recording, the responses of D. suzukii females to three reference stimuli (dichloromethane solvent, 1-hexanol and 2-hexanal) were recorded. These reference compounds are well known plant volatiles and significantly elicited antennal responses in D. suzukii [35].

Each stimulus was prepared by absorbing $25 \mu \mathrm{L}$ of dichloromethane solution at a concentration of $1 \mu \mathrm{g} / \mu \mathrm{L}$ on the filter paper. A glass capillary indifferent electrode filled with Kaissling solution was introduced into the detached fly's head. The different electrode was a similar capillary, connected to the distal end of antenna. Thereafter, the stimulus output was directly delivered to the antenna with the Pasteur pipette through the air flow, activated by operating an external pedal linked to the stimulus air control. Each stimulus pipette was replaced after three consecutive recordings.

\subsection{Data Analysis}

In the preliminary field assessment of DD food bait attractiveness, the mean weekly number and percentages of D. suzukii captures in each trap were obtained on the $\log / \operatorname{logit}$ scale along with associated 95\% confidence limits: these were back-transformed then divided by the number of days since trial set-up, to obtain the mean number of catches per trap per day. All analyses were carried out with Genstat [36]. 
The data obtained from the experimental work for the evaluation of the performance of $O$. oeni strains were analyzed by calculating the difference in the mean absorbance of bacterial growth. EAG recordings were analyzed by EAG software (EAG 2000 version 2.7, Syntech, Hilversum, Netherlands), and evaluated by measuring the maximum amplitude of negative deflection (-mVolt) obtained by stimulating the antenna. EAG amplitude values were compared across treatments by means of parametric one-way ANOVA, followed by Tukey's test for the post hoc comparison of means. Homogeneity of variance had been previously determined with the Levene's test. The statistical analyses were performed using Statistica, version 9 (Statsoft Inc., Tulsa, Oklahoma).

\section{Results}

3.1. Field Assessment of the Attractiveness of Droskidrink ${ }^{\circledR}$ Food Baits Inoculated with Different Lactic Acid Bacteria

Figure 1 shows the total number of $D$. suzukii flies captured by different bait types during the preliminary field experiment. Generally, DD baits inoculated with the selected LAB species caught a significantly higher number of flies in comparison to the other DD baits $(p<0.001)$. Bait type A caught a significantly higher number of $D$. suzukii adults than baits inoculated with Lactobacillus spp. and Pediococcus spp. In three trials, there were significant differences in the numbers of males and total number of $D$. suzukii between the treatments $(p=0.027,0.015$, and 0.003 for males and $p=0.027,0.017$, and 0.001 for total $D$. suzukii for trials 1, 2, and 3, respectively) (Figure 1).

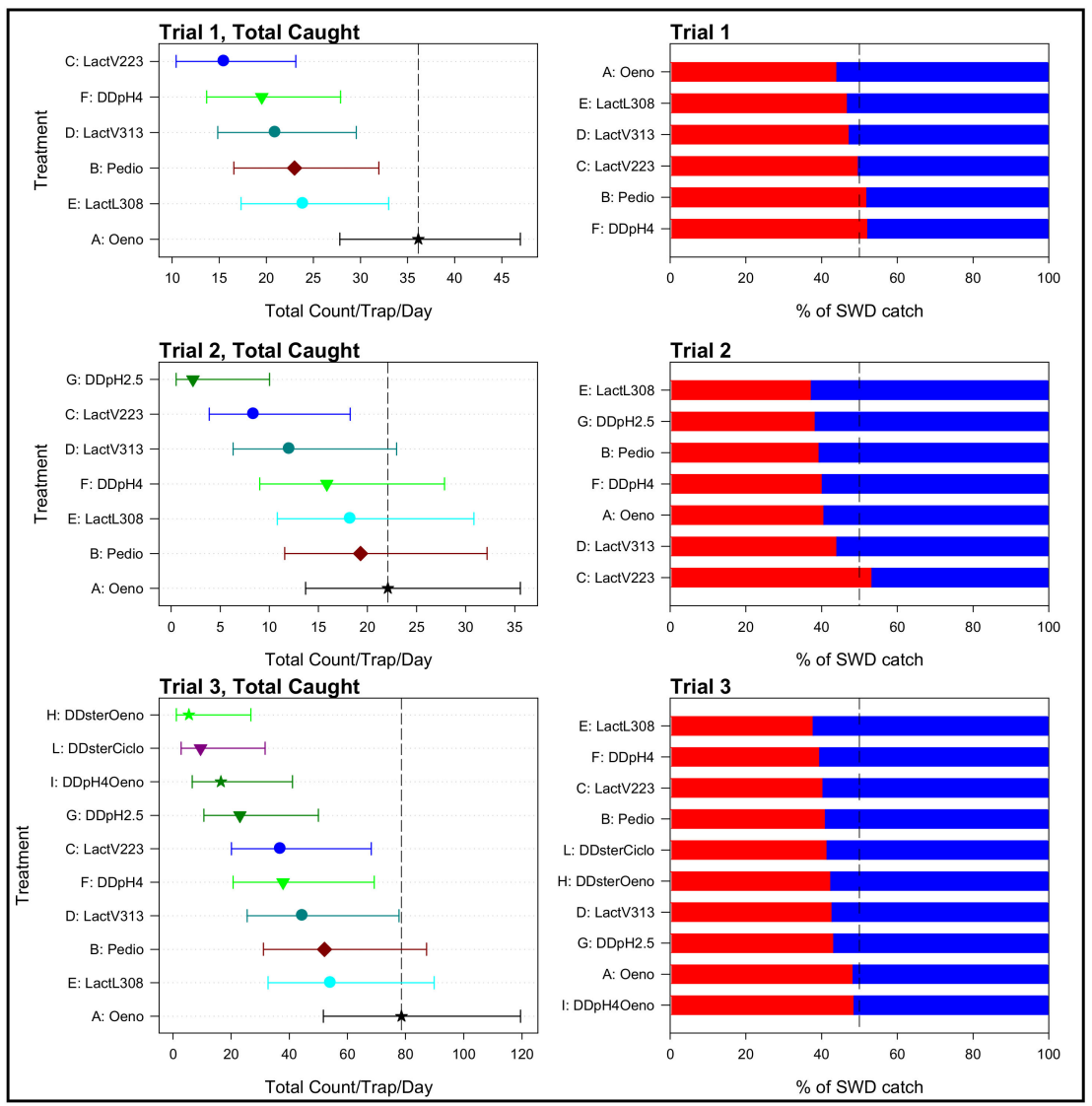

Figure 1. Left: Mean total SWD caught per trap per day for the three trials (including the final assessment, trial 3), with treatment in increasing order of the mean. Error bars are $95 \%$ confidence limits, and vertical dashed line is at the mean fortreatment A. Right: percentage of the total SWD that were female or male, for each of the three trials. Treatments are in increasing order of the $\%$ female. Dashed vertical line is at $50 \%$. The red color indicates the percentage of caught females and the blue color indicates the percentage of caught males. 
There was no significant difference in the numbers of females for trial $1(p=0.236)$, but there were significant differences in trials 2 and 3 ( $p=0.023,0.001$, respectively). The percentage of female D. suzukii was similar for all treatments in trials 1 and 3 ( $p=0.828$, 0.061 , respectively), but there was a significant difference between treatments in trial $2(p$ $=0.039$ ). Generally, the highest capture levels in all treatments were obtained in the fifth week of the experimental period and the capture peak corresponded to bait A.

The mean numbers of females were strongly correlated with the mean numbers of males ( $r=0.95,0.96,0.95$ for the three trials, respectively), reflecting that the patterns between the means were very similar for both sexes, and thus also similar to patterns in the total number of D. suzukii (Figure 1). For all three trials, the highest total number of D. suzukii was associated with bait A. Among the six baits tested first, bait type $C$ had the lowest total number of $D$. suzukii, followed by bait type F. However, the total number of $D$. suzukii in bait type $\mathrm{G}$ was lower than in $\mathrm{C}$, and for trial 3, the total number of D. suzukii in baits $\mathrm{H}$, I, and L was lower than in bait G (Table S1).

\subsection{Assessment of the Performance of Oenococcus oeni Strains in Droskidrink ${ }^{\circledR}$ Food Bait}

All O. oeni strains, showed different growth rates in MRSm, with respect to the standard strains employed in the preliminary field experiment. The four considered variables ( $\mathrm{pH}$ value 4.0, acetic acid concentration $45 \mathrm{~g} / \mathrm{L}$, ethanol content $4 \%(v / v)$, and incubation temperature $15^{\circ} \mathrm{C}$ ) allowed reasonable sorting of the $\mathrm{O}$. oeni strains, according to the resistance to such limiting parameters.

Figure 2 shows the difference in the mean absorbance of $O$. oeni strains growth. Strain 3 showed the highest tolerance to low $\mathrm{pH}(4.0)$, and strain 14 had a significant tolerance to high acetic acid concentration $(45 \mathrm{~g} / \mathrm{L})$. When compared with the control, strain 3 and strain 14 exhibited higher growth than the control at the tested $\mathrm{pH}$ and acetic acid concentration. Likewise, strain 2 showed the highest growth at high ethanol content (4\%) and strain 12 showed the highest growth at low temperature $\left(15^{\circ} \mathrm{C}\right)$. In comparison with the control, growth of strain 2 and strain 12 was higher than the control at the tested ethanol content and temperature. Strain 2 and strain 12 showed high tolerance to high ethanol content (4\%) and low temperature $\left(15^{\circ} \mathrm{C}\right)$, respectively. Overall, strain 2 and strain 12 showed a significant tolerance to the main limiting parameters for $O$. oeni growth, i.e., high ethanol content and low temperature. Strain 13 showed the highest tolerance to three limiting parameters, i.e., low $\mathrm{pH}$, high acetic acid concentration, and low temperature (Table S2). Thus, the three strains selected for further experimentation for the evaluation of the behavioral responses of D. suzukii to volatiles emitted during fermentation process were strain 2 , strain 12 , and strain 13.

\subsection{Electroantennography Responses of Drosophila suzukii Females to Volatile Collection of Oenococcus oeni Strains in Droskidrink ${ }^{\circledR}$ Food Bait}

The EAG responses of female D. suzukii to the headspace extracts obtained from DD inoculated with O. oeni strains are depicted in Figure 3. The reference compounds, 1-hexanol and 2-hexanal, elicited strong antennal responses (Figure S2). There were no statistical differences between the samples (DD, strain 2,12 and 13) and the reference strain (MRI 10000), but all baits were statistically different from both the solvents and the blank control (ANOVA: d.f. $=47 ; \mathrm{F}=8.6 ; p<0.001$ ) (Table S3). 


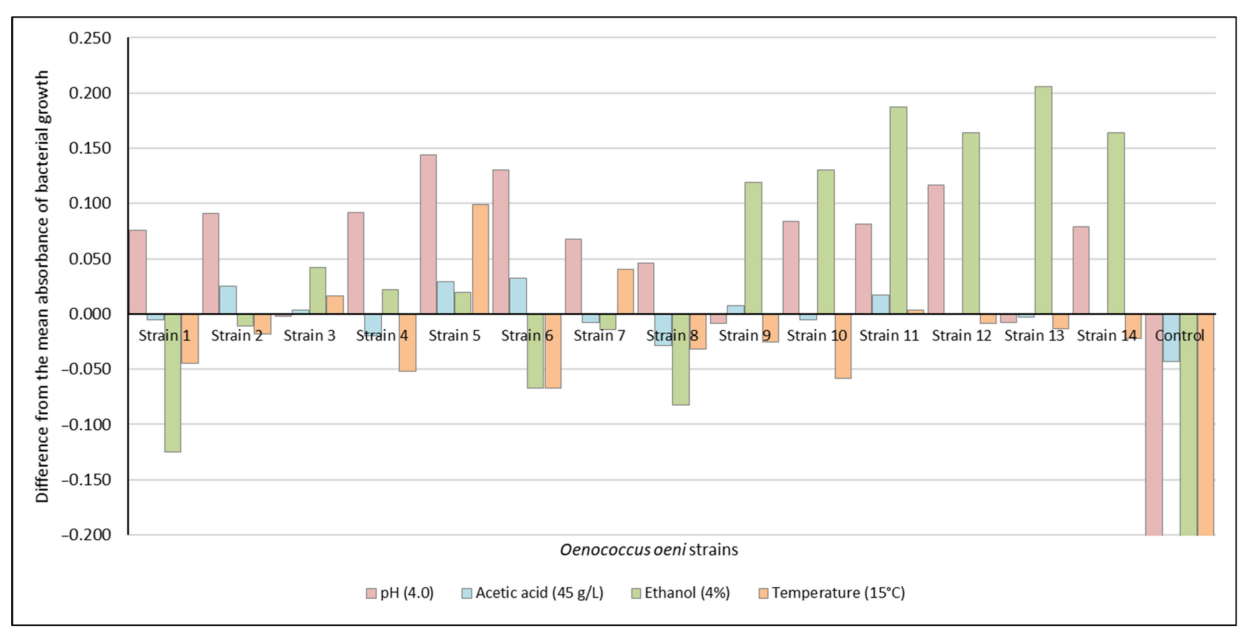

Figure 2. Differences in the mean absorbance of bacterial growth in synthetic media considering the four main DD limiting factors for O. oeni growth ( $\mathrm{pH} 4.0)$, ethanol (4\%), acetic acid (45 g/L), and temperature $\left(15^{\circ} \mathrm{C}\right)$.

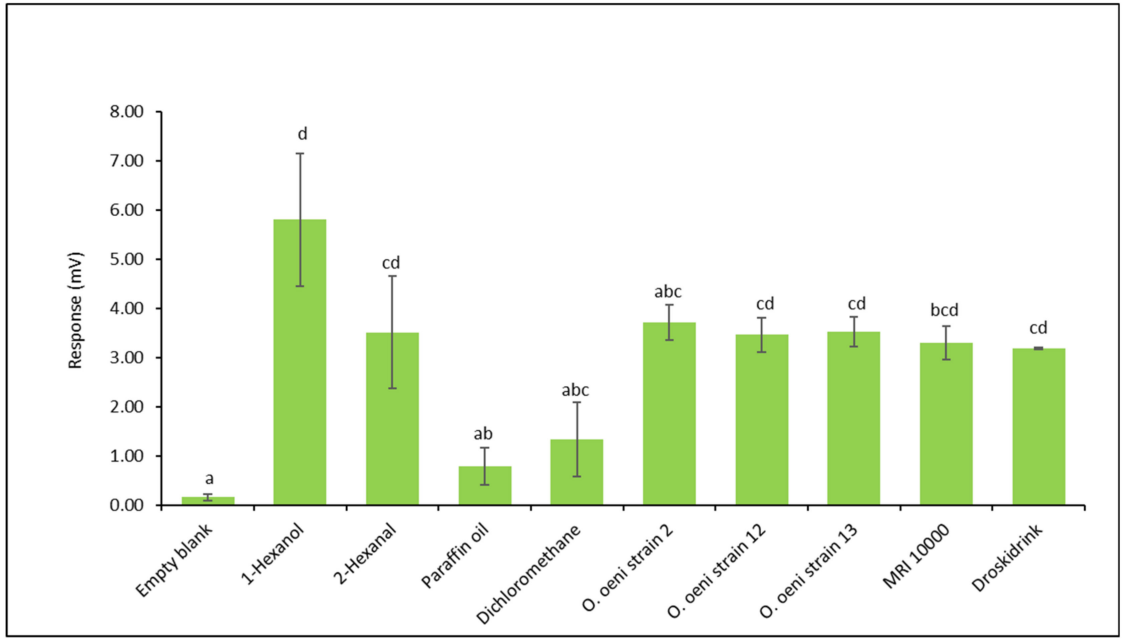

Figure 3. Mean electroantennography (EAG) responses $(\mathrm{mV})$ of $D$. suzukii mated female antennae elicited by commercial DD and DD inoculated with different $O$. oeni strain 2, strain 12 and strain 13. Control stimuli: empty blank, paraffin oil, and dichloromethane solvent. Reference compounds: 1-hexanol, 2-hexanal. Baits: commercial DD, O. oeni reference strain (MRI 10000), strain 2, strain 12, and strain 13. The standard deviation of the means is reported. Bars represented by similar letters are significantly indifferent $(p>0.05)$.

\section{Discussion}

The attractive effect of selected LAB species related to the biochemical changes of the bait such as the increase in the standard $\mathrm{pH}$ of DD (typically about 2.5) up to 4.0, since this value is recognized as ideal for the hetero-fermentative activity of wine LAB $[37,38]$ was explored. Overall, the DD baits inoculated with the different LAB species attracted a considerably higher number of flies in comparison to other baits. The highest attractiveness was observed in the bait inoculated with O. oeni, which coincides with the outcomes of recent research comparing different lures for their attractiveness for D. suzukii in sweet cherry orchards [20]. On the contrary, pasteurized DD baits and DD bait mixed with tetracycline did not show any considerable attractiveness due to limited microbial activity in the liquid bait.

Our findings imply that the utilization of the selected LAB species in DD bait was carried out successfully in the preliminary field trials. This is not surprising considering 
that they are an extremely important group of industrially related lactic bacteria, and considering their behavior and robustness under stressful conditions. LAB strains were used in our study to ferment the liquid bait and produce bioactive volatile compounds that are associated with D. suzukii attraction. Therefore, the type and concentration of volatiles present in the DD bait are greatly affected by the LAB strain used. Concerning the different species of $L A B$, it has been already mentioned that $O$. oeni have a remarkable hetero-fermentative activity and resistance in a low $\mathrm{pH}$ environment $[37,38]$. Therefore, their enhanced biological activity and effectiveness in trapping D. suzukii is expected. Recently, the addition of O. oeni to DD bait was evaluated in a comparative survey using Droso-Trap for monitoring fly populations in sweet cherry orchards. It was found that DD with $O$. oeni was effective in attracting D. suzukii during the blooming and until the beginning of fruit ripening [20]. These findings support our results, which show that $O$. oeni contributed to the highest attractiveness of the DD baits. Despite the differences in DD composition, field temperature, fruit phenological phases and pest population density between this comparative survey and our study, O. oeni in DD showed a similar trend in attracting flies, indicating a consistent fly response towards O. oeni.

Furthermore, the obtained results show that the $\mathrm{pH}$ value in the liquid bait is a key point for the improvement of trap efficacy. In fact, the standard value of $\mathrm{pH}$ in $\mathrm{DD}$, around 2.5 , is unsuitable for the development of most LAB species, and thus the $\mathrm{pH}$ level has to be increased in order to provide them with an appropriate growing environment. On the other hand, too high $\mathrm{pH}$ values would promote a non-selective growth of contaminant microbes (yeasts, bacteria, or mold), causing a rapid depletion of the nutrients contained in the bait.

Likewise, oenological trials showed that the $\mathrm{pH}$ value, adopted in the experiment, induces the shift of the metabolic activity of LAB from homo- to hetero-fermentation of sugars, increasing their energetic yield $[37,38]$. In our study, the selected LAB species were isolated from traditional Italian wine, indicating their ability as commercial starters to tolerate the stress conditions, i.e., acidic $\mathrm{pH}$, encountered in the industrial winemaking process. However, several studies reported the ability of wine LAB to develop stress-induced responses through various mechanisms $[39,40]$. In earlier studies, O. oeni strains showed a high adaptive behavior to an acidic environment and a good malolactic performance at pH 3.5 and 3.0, respectively [31,40]. Similarly, Lactobacillus spp. and Pediococcus spp. exhibited acidophilic behavior at pH 3.0 and 3.5, respectively [41,42]. Under such stress conditions, the Oenococcus, Lactobacillus, and Pediococcus genera use the malolactic fermentation pathway to convert malic acid to lactic acid with the production of $\mathrm{CO}_{2}$. This pathway contributes to LAB survival enhancement and promotes growth [40]. In addition, it enhances their aroma profile, leading to a prevalence of the fruity aroma over the vegetative ones [43]. Based on the previous findings, it is obvious that the LAB tested in our study were able to tolerate low $\mathrm{pH}$ and to produce fermentative volatiles that mediate fly attraction and significantly contribute to improving the attractiveness of the DD bait. Among all the tested LAB, O. oeni with DD was the most attractive bait. Overall variations in capturing levels between the tested $\mathrm{LAB}$ could be related to environmental conditions that may affect the fermentation process and, consequently, the volatile profiles qualitatively and quantitatively. The non-linear increase in trap catches over the time of field exposure could be explained by the fact that the traps were only refilled every week to compensate the bait evaporation and hence the liquid bait was not completely replaced during the entire duration of the field test in order to maintain the original population of bacteria inside it.

In our field trials, it was observed that DD baits with LAB attracted D. suzukii flies in a species- and strain-dependent manner in the first week. The following three weeks showed a fluctuation and a decrease in captured flies. Thereafter, capture peaks were found in all DD baits with LAB in the fifth week. These sharp variations in the weekly captures can be related to several factors affecting the LAB metabolic activities. The high concentration of LAB $\left(10^{6} \mathrm{CFU} / \mathrm{mL}\right)$ in the first week may help to maintain their metabolic activities and production of volatiles that mediate fly's attraction [44]. In the subsequent weeks, it is 
anticipated that stress conditions in DD bait have a negative impact on LAB growth and metabolism, resulting in low attraction levels. As part of the stress response, LAB develop an adaptive response which enables them to survive and maintain their metabolic activities. During the fermentation process, the LAB can produce a series of biologically active compounds that affect fruit flies [45]. Consequently, volatile compounds that are most prevalent in the main components of DD (wine and vinegar), i.e., methanol, ethanol, acetic acid, and ethyl acetate, will be enriched by LAB fermentation. The results and observations reported in our study clearly suggest that the tested LAB strains contribute to improving the attractiveness of a commercial lure (DD) and recognize them as suitable agents for a more effective lure. O. oeni, the best-adapted species to the stress conditions [40], can be exploited as pest control agent in eco-friendly and sustainable management strategies.

In the laboratory evaluation of O. oeni performance in DD conditions, the addition of bacteria to $\mathrm{DD}$ as bio-catalyzers for the production of bioactive volatile compounds is completely new. Therefore, it is necessary to evaluate the performance of $O$. oeni strains under stress conditions in DD bait. The four main DD characteristics (low $\mathrm{pH}$, high ethanol content, high acetic acid concentration, and low temperatures during field application) that can limit the growth of $O$. oeni strains were tested. The outcomes are particularly interesting in view of the double effect of acetic acid on experimental conditions. In addition, O. oeni was able to lower the $\mathrm{pH}$ level (2.5), as an end-product of many of its metabolic activities [37], and it can inhibit bacterial activity, affecting the ratio between substrates consumption and metabolites production.

The laboratory tests made it possible to identify three O. oeni strains that exhibited different adaptation to the peculiar DD composition. These strains were tested for their ability to produce volatile compounds, by the fermentative metabolism in DD, with a biological activity on $D$. suzukii. However, the relevant difference among the three $O$. oeni strains required further field tests in order to select the most attractive bait for D. suzukii monitoring.

In EAG analysis, 1-hexanol was already detected among the volatile bouquet of fresh mature D. suzukii host fruits and elicited fly's antennal responses in gas chromatographic analysis coupled with electroantennographic detection experiments (GC-EAD) [35,46]. Moreover, EAG analysis confirmed remarkable insect sensitivity to both DD and DD inoculated with O. oeni. Additional GC-EAD experiments were performed [47] to understand which single compounds can be perceived by the olfactory system of D. suzukii. The selection of single biologically active compounds may pave the way to the development of synthetic attractants.

To better explain D. suzukii preference for DD baits inoculated with O. oeni strains, a recent work identified the volatiles profile of the various strains and determines their essential role in fly's preferences [47]. Although it was not possible to identify an overall attraction pattern for O. oeni strains, the study showed that three O. oeni strains produced the most attractive volatiles. A combination of the most effective strains was used to optimize trap efficiency for pest management. To get a deeper insight into their nature, fly responses to the new DD baits using different trap designs were determined in open field conditions [47]. Such field trials will be exploited to develop a new, efficient trapping system for $D$. suzukii management.

\section{Conclusions}

The study demonstrates that different strains of the O. oeni species can improve the attractiveness of the commercial food bait DD, making it a powerful tool for pest control and monitoring. The outcomes of a series of preliminary field trials and laboratory tests highlight the importance of further investigations of the chemical and microbiological properties of DD with regard to the mechanisms of fly's attraction, in order to produce a highly attractive bait for pest management.

Additionally, the findings of this study pave the way for the development of a new trapping concept, in which the attractiveness of DD would be improved by the use of 
LAB volatiles in a strain-dependent manner. Thus, further laboratory and open field trials on the chemical characterization of volatiles produced in baits inoculated with $O$. oeni strains, providing the optimal bacterial growth in open field conditions, are useful for the successful setup of new efficient traps.

Supplementary Materials: The following are available online at https: / www.mdpi.com/2075-445 0/12/2/153/s1, Figure S1: Layout and duration of field trials, Figure S2: Electroantennogram plots recorded from SWD antenna, in response to volatile collection of O. oeni strains in Droskidrink ${ }^{\circledR}$ Bait., Table S1: Field assessment of attractiveness of Droskidrink ${ }^{\circledR}$ baits inoculated with lactic acid bacteria, Table S2: Assessment of O. oeni strains' growth in Droskidrink ${ }^{\circledR}$ bait, Table S3: Electroantennography responses of SWD females to volatile collection of O. oeni strains in Droskidrink ${ }^{\circledR}$ Bait.

Author Contributions: The study was conceived by G.A. and A.D.C. The experiments were conducted by G.M. and R.G. under supervision of A.D.C. and G.A. The statistical analysis was conducted by R.G., G.Đ., and G.A. The manuscript was drafted by A.A. and M.M.H. and revised by G.Đ., S.G., and G.A. The funding acquisition was provided by F.W. All authors have read and agreed to the published version of the manuscript.

Funding: This project has received funding from the European Union's Horizon 2020 research and innovation programme under the Marie Skłodowska-Curie grant agreement no. 722642 (INTERFUTURE).

Institutional Review Board Statement: Not applicable.

Informed Consent Statement: Not applicable.

Data Availability Statement: The data presented in this study are available on request from the corresponding author.

Acknowledgments: The authors would like to thank Ruth C. Butler (The New Zealand Institute for Plant and Food Research Limited) for providing the statistical support. We would also like to thank Noureddine Besselma (Lincoln University, New Zealand) for his support in English editing.

Conflicts of Interest: The authors declare no conflict of interest. The funders had no role in the design of the study; in the collection, analyses, or interpretation of data; in the writing of the manuscript, or in the decision to publish the results.

\section{References}

1. Lee, J.C.; Bruck, D.J.; Dreves, A.J.; Ioriatti, C.; Vogt, H.; Baufeld, P. In Focus: Spotted wing drosophila, Drosophila suzukii, across perspectives. Pest Manag. Sci. 2011, 67, 1349-1351. [CrossRef]

2. Cini, A.; Ioriatti, C.; Anfora, G. A review of the invasion of Drosophila suzukii in Europe and a draft research agenda for integrated pest management. Bull. Insectol. 2012, 65, 149-160.

3. Asplen, M.K.; Anfora, G.; Biondi, A.; Choi, D.S.; Chu, D.; Daane, K.M.; Gibert, P.; Gutierrez, A.P.; Hoelmer, K.A.; Hutchison, W.D.; et al. Invasion biology of spotted wing Drosophila (Drosophila suzukii): A global perspective and future priorities. J. Pest Sci. 2015, 88, 469-494. [CrossRef]

4. Rota-Stabelli, O.; Blaxter, M.; Anfora, G. Drosophila suzukii. Curr. Biol. 2013, 23, R8. [CrossRef]

5. Pavlova, A.K.; Dahlmann, M.; Hauck, M.; Reineke, A. Laboratory bioassays with three different substrates to test the efficacy of insecticides against various stages of Drosophila suzukii (Diptera: Drosophilidae). J. Insect Sci. 2017, 17. [CrossRef] [PubMed]

6. Cahenzli, F.; Strack, T.; Daniel, C. Screening of 25 different natural crop protection products against Drosophila suzukii. J. Appl. Entomol. 2018, 142, 563-577. [CrossRef]

7. Shawer, R.; Tonina, L.; Tirello, P.; Duso, C.; Mori, N. Laboratory and field trials to identify effective chemical control strategies for integrated management of Drosophila suzukii in European cherry orchards. Crop Prot. 2018, 103, 73-80. [CrossRef]

8. Rice, K.B.; Short, B.D.; Leskey, T.C. Development of an attract-and-kill strategy for Drosophila suzukii (Diptera: Drosophilidae): Evaluation of attracticidal spheres under laboratory and field conditions. J. Econ. Entomol. 2017, 110, 535-542. [CrossRef] [PubMed]

9. Spies, J.M.; Liburd, O.E. Comparison of attractants, insecticides, and mass trapping for managing Drosophila suzukii (Diptera: Drosophilidae) in Blueberries. Fla. Entomol. 2019, 102, 315-321. [CrossRef]

10. Rossi-Stacconi, M.V.; Grassi, A.; Dalton, D.T.; Miller, B.; Ouantar, M.; Loni, A.; Grassi, A.; Gottardello, A.; Rota-Stabelli, O.; Anfora, G. First field records of Pachycrepoideus vindemiae as a parasitoid of Drosophila suzukii in European and Oregon small fruit production areas. Entomologia 2013, 1, 11-16. [CrossRef]

11. Cuthbertson, A.G.S.; Blackburn, L.F.; Audsley, N. Efficacy of commercially available invertebrate predators against Drosophila suzukii. Insects 2014, 5, 952-960. [CrossRef] 
12. Girod, P.; Rossignaud, L.; Haye, T.; Kenis, M. First results of the testing of Asian parasitoids as potential biological control agents of the Spotted Wing Drosophila, Drosophila suzukii. IOBC-WPRS Bull. 2017, 123, 186-187.

13. Ibouh, K.; Oreste, M.; Bubici, G.; Tarasco, E.; Rossi-Stacconi, M.V.; Ioriatti, C.; Verrastro, V.; Anfora, G.; Baser, N. Biological control of Drosophila suzukii: Efficacy of parasitoids, entomopathogenic fungi, nematodes and deterrents of oviposition in laboratory assays. Crop Prot. 2019, 125. [CrossRef]

14. Lasa, R.; Tadeo, E.; Toledo-Hérnandez, R.A.; Carmona, L.; Lima, I.; Williams, T. Improved capture of Drosophila suzukii by a trap baited with two attractants in the same device. PLOS ONE 2017, 12, 1-19. [CrossRef] [PubMed]

15. Baroffio, C.A.; Richoz, P.; Fischer, S.; Kuske, S.; Linder, C.; Kehrli, P. Monitoring Drosophila suzukii in Switzerland in 2012. J. Berry Res. 2014, 4, 47-52. [CrossRef]

16. Iglesias, L.E.; Nyoike, T.W.; Liburd, O.E. Effect of trap design, bait type, and age on captures of Drosophila suzukii (Diptera: Drosophilidae) in berry crops. J. Econ. Entomol. 2014, 107, 1508-1518. [CrossRef] [PubMed]

17. Vaccari, G.; Caruso, S.; Nouhaud, C.; Maistrello, L. Comparison of different trap types for Drosophila suzukii in cherry fields. IOBC-WPRS Bull. 2015, 109, 211-214.

18. Mazzetto, F.; Pansa, M.G.; Ingegno, B.L.; Tavella, L.; Alma, A. Monitoring of the exotic fly Drosophila suzukii in stone, pome and soft fruit orchards in NW Italy. J. Asia Pac. Entomol. 2015, 18, 321-329. [CrossRef]

19. Cha, D.H.; Loeb, G.M.; Linn, C.E.; Hesler, S.P.; Landolt, P.J. A Multiple-choice bioassay approach for rapid screening of key attractant volatiles. Environ. Entomol. 2018, 47, 946-950. [CrossRef] [PubMed]

20. Tonina, L.; Grassi, A.; Caruso, S.; Mori, N.; Gottardello, A.; Anfora, G.; Giomi, F.; Vaccari, G.; Ioriatti, C. Comparison of attractants for monitoring Drosophila suzukii in sweet cherry orchards in Italy. J. Appl. Entomol. 2018, 142, 18-25. [CrossRef]

21. Clymans, R.; Van Kerckvoorde, V.; Bangels, E.; Akkermans, W.; Alhmedi, A.; De Clercq, P.; Beliën, T.; Bylemans, D. Olfactory preference of Drosophila suzukii shifts between fruit and fermentation cues over the season: Effects of physiological status. Insects 2019, 10, 200. [CrossRef]

22. Mazzetto, F.; Gonella, E.; Crotti, E.; Vacchini, V.; Syrpas, M.; Pontini, M.; Mangelinckx, S.; Daffonchio, D.; Alma, A. Olfactory attraction of Drosophila suzukii by symbiotic acetic acid bacteria. J. Pest Sci. 2016, 89, 783-792. [CrossRef]

23. Hayek, A.S.; Ibrahim, A.S. Current limitations and challenges with lactic acid bacteria: A review. Food Nutr. Sci. 2013, 73-87. [CrossRef]

24. Hoefnagel, M.H.N.; Starrenburg, M.J.C.; Martens, D.E.; Hugenholtz, J.; Kleerebezem, M.; Van Swam, I.I.; Bongers, R.; Westerhoff, H.V.; Snoep, J.L. Metabolic engineering of lactic acid bacteria, the combined approach: Kinetic modelling, metabolic control and experimental analysis. Microbiology 2002, 148, 1003-1013. [CrossRef]

25. Endo, A.; Shintaro, M.; Yasuhiro, T.; Wolfgang, K.; Masanori, A.; Leon, D.; Salminenf, S. Fructophilic lactic acid bacteria, a unique group of fructose-fermenting microbes. Appl. Environ. Microbiol. 2018, 84, 1-14. [CrossRef]

26. Capozzi, V.; Russo, P.; Beneduce, L.; Weidmann, S.; Grieco, F.; Guzzo, J.; Spano, G. Technological properties of Oenococcus oeni strains isolated from typical southern Italian wines. Lett. Appl. Microbiol. 2010, 50, 327-334. [CrossRef]

27. Venu, I.; Durisko, Z.; Xu, J.; Dukas, R. Social attraction mediated by fruit flies' microbiome. J. Exp. Biol. 2014, $217,1346-1352$. [CrossRef] [PubMed]

28. Qiao, H.; Keesey, I.W.; Hansson, B.S.; Knaden, M. Gut microbiota affects development and olfactory behavior in Drosophila melanogaster. J. Exp. Biol. 2019, 222. [CrossRef]

29. Vacchini, V.F. Microbial Ecology of the Spotted Wing Fly Drosophila suzukii. Ph.D. Thesis, Università Degli Studi di Milano, Milano, Italy, 2014. [CrossRef]

30. Grassi, A.; Anfora, G.; Maistri, S.; Maddalena, G.; De Gristofaro, A.; Savini, G.; Savini, G.; Ioriatti, C. Development and efficacy of Droskidrink, a food bait for trapping Drosophila suzukii. IOBC-WPRS Bull. 2015, 109, 26-28.

31. Solieri, L.; Genova, F.; De Paola, M.; Giudici, P. Characterization and technological properties of Oenococcus oeni strains from wine spontaneous malolactic fermentations: A framework for selection of new starter cultures. J. Appl. Microbiol. 2010, 108, 285-298. [CrossRef]

32. McCullagh, P.; Nelder, J.A. Generalized Linear Models, 2nd ed.; Chapman \& Hall: London, UK, 1989; p. 511.

33. Guzzon, R.; Poznanski, E.; Conterno, L.; Vagnoli, P.; Krieger-Weber, S.; Cavazza, A. Selection of a new highly resistant strain for malolactic fermentation under difficult conditions. S. Afr. J. Enol. Vitic. 2009, 30, 133-141. [CrossRef]

34. Salvagnin, U.; Malnoy, M.; Thöming, G.; Tasin, M.; Carlin, S.; Martens, S.; Vrhovsek, U.; Angeli, S.; Anfora, G. Adjusting the scent ratio: Using genetically modified Vitis vinifera plants to manipulate European grapevine moth behaviour. Plant Biotechnol. J. 2018, 16, 264-271. [CrossRef] [PubMed]

35. Revadi, S.; Vitagliano, S.; Rossi-Stacconi, M.V.; Ramasamy, S.; Mansourian, S.; Carlin, S.; Vrhovsek, U.; Becher, P.G.; Mazzoni, V.; Rota-Stabelli, O.; et al. Olfactory responses of Drosophila suzukii females to host plant volatiles. Physiol. Entomol. 2015, 40, 54-64. [CrossRef]

36. Payne, R.; Murray, D.; Baird, D. The Guide to the Genstat Command Language (Release 19); VSN International, Hemel Hempsted: Hertfordshire, UK, 2017.

37. Lonvaud-Funel, A. Biogenic amines in wines: Role of lactic acid bacteria. FEMS Microbiol. Lett. 2001, 199, 9-13. [CrossRef]

38. Liu, S.Q. Malolactic fermentation in wine-beyond deacidification. J. Appl. Microbiol. 2002, 92, 589-601. [CrossRef]

39. Spano, G.; Massa, S. Environmental stress response in wine lactic acid bacteria: Beyond Bacillus subtilis. Crit. Rev. Microbiol. 2006, 32, 77-86. [CrossRef] 
40. Papadimitriou, K.; Alegría, Á.; Bron, P.A.; de Angelis, M.; Gobbetti, M.; Kleerebezem, M.; Lemos, J.A.; Linares, D.M.; Ross, P.; Stanton, C.; et al. Stress Physiology of Lactic Acid Bacteria. Microbiol. Mol. Biol. Rev. 2016, 80, 837-890. [CrossRef]

41. Succi, M.; Pannella, G.; Tremonte, P.; Tipaldi, L.; Coppola, R.; Iorizzo, M.; Lombardi, S.J.; Sorrentino, E. Sub-optimal pH preadaptation improves the survival of Lactobacillus plantarum strains and the malic acid consumption in wine-like medium. Front. Microbiol. 2017, 8, 1-12. [CrossRef]

42. Nisiotou, A.A.; Dourou, D.; Filippousi, M.E.; Diamantea, E.; Fragkoulis, P.; Tassou, C.; Banilas, G. Genetic and technological characterisation of vineyard- and winery-associated lactic acid bacteria. BioMed Res. Int. 2015, 1-8. [CrossRef]

43. Lerm, E.; Engelbrecht, L.; du Toit, M. Malolactic fermentation: The ABC's of MLF. S. Afr. J. Enol. Vitic. 2010, 31, 186-212. [CrossRef]

44. Di Cagno, R.; Coda, R.; De Angelis, M.; Gobbetti, M. Exploitation of vegetables and fruits through lactic acid fermentation. Food Microbiol. 2013, 33, 1-10. [CrossRef]

45. Schulz, S.; Dickschat, J.S. Bacterial volatiles: The smell of small organisms. Nat. Prod. Rep. 2007, 24, 814-842. [CrossRef]

46. Abraham, J.; Zhang, A.; Angeli, S.; Abubeker, S.; Michel, C.; Feng, Y.; Rodriguez-Saona, C. Behavioral and antennal responses of Drosophila suzukii (Diptera: Drosophilidae) to volatiles from fruit extracts. Environ. Entomol. 2015, 44, 356-367. [CrossRef] [PubMed]

47. Đurović, D.; Alawamleh, A.; Carlin, S.; Maddalena, G.; Guzzon, R.; Mazzoni, V.; Dalton, D.T.; Walton, V.M.; Suckling, D.M.; Butler, R.C.; et al. Liquid Baits with Oenococcus oeni increase captures of Drosophila suzukii. Insects 2021, 12, 66. [CrossRef] [PubMed] 